\title{
Shack-Hartmann tomographic wavefront reconstruction using LGS: analysis of spot elongation and fratricide effect
}

\author{
Clélia Robert $^{1, a}$, Jean-Marc Conan ${ }^{1}$, Damien Gratadour ${ }^{2}$, Cyril Petit $^{1}$, and Thierry Fusco ${ }^{1}$ \\ 1 Office National d'Études et de Recherches Aérospatiales, BP 72, 92322 Châtillon, France \\ 2 LESIA, Observatoire de Paris, CNRS, UPMC, Université Paris Diderot; 5 Place Jules Janssen, \\ 92190 Meudon, France
}

\begin{abstract}
Noise effects induced by elongation have to be considered globally in a multi-channel tomographic reconstruction analysis. Such an analysis allows a fine estimation of performance and to compare launching options. We developed a modal LGS tomographic code based on pseudo-analytical matrix formalism. This modal analysis is computing and memory intensive. Therefore we limit the simulation to downscaled cases with diameters ranging from $\mathrm{D}=4$ to $21 \mathrm{~m}$. We also limit the number of turbulent layers to a few equivalent layers. Including spot elongation and Rayleigh fratricide effect, when considering a global tomographic reconstruction, edge launching gives lightly better performance and limits the risk of possible underestimation of fratricide scatter.
\end{abstract}

\section{Introduction}

Present projects of Extremely Large Telescopes (ELT) rely on wide-field-of-view adaptive optics systems such as multi-conjugate AO, multi-object AO, ground-layer AO and Laser Tomography AO. All these systems will use atmospheric tomography to reconstruct the turbulent phase volume using different constellations of natural (NGS) and laser guide stars (LGS). A laser guide star is not only at a finite distance leading to cone effect, but also extended over the whole thickness of the sodium layer. A Shack-Hartmann WFS (SH WFS) looking at a LGS is confronted to spot elongation leading to specific slope noise [1] with possibly anisoplanatism [2,5]. Systems using multiple LGS beams with CW lasers are also affected by fratricide effect. A SH WFS looking at a LGS may collect the Rayleigh scattered light of other LGSs, causing a background increase in some subapertures. These perspective and fratricide effects are launching dependent. In this paper, we explore the interactions between the spot elongation and fratricide effects, with analytical tomographic modal wavefront reconstruction techniques to eventually define a preferred launching scheme. This is a generalization of the noise propagation of the work presented in [1] to a multi-LGS constellation sensed through a multi-layer turbulence profile supplementing the work done in [3]. We envisage the impact of these effects in terms of SH WFS accuracy and related performance on central direction in the scientific field of view (FoV) for MAORY/ATLAS/EAGLE projects [6-8].

\section{Reconstruction methods and modelling}

The code we developed is able to quantify the impact of LGS noise on tomographic wavefront reconstruction. To sum up the code is a matrix-based simulation tool using analytical formulae of Weighted Center of Gravity (WCoG) for centroiding to compute tomography + noise wavefront error on a modal basis. The Shack-Hartmann wavefront reconstruction takes into account:

- Spot elongation related to the laser launching option

- Additive fratricide background

- Various spot position estimators (WCoG, possibly correlation)

- Loss of slope data due to the spider

- Regularisation using noise correlation and turbulent priors

a e-mail: clelia.robert@onera.fr

This is an Open Access article distributed under the terms of the Creative Commons Attribution-Noncommercial License, which permits unrestricted use, distribution, and reproduction in any noncommercial medium, provided the original work is properly cited. 
The reconstruction methods of the wavefront are based on the linear measurement equation representing the SH slopes $s$ with noise $n$ :

$$
s=D \phi+n
$$

The wavefront $\phi$ is decomposed on Karhunen Loeve basis (KL). We implement several methods to reconstruct it: Least Squares (LS), Weighted Least Squares (WLS), MAP with and without uniform noise (UNIFMAP and MAP resp.) similarly to the formalism described in [3]. We will use mainly the MAP, although we started to compare the four methods in a down-scaled case presented in section 2.3. All these methods are matrix-multiply combinations using the LGS noise covariance matrix $C_{n}$ due to elongation and possibly priors on turbulence (via $C_{\phi}$ ) especially for MAP methods. The interaction matrix D is calculated by multiplying the SH WFS matrix with a projection matrix which accounts for the different directions $k$ of the LGS in the FoV where the turbulent volume is probed. The phase reconstructed in a multi-LGS scheme is written as

$$
\phi=\left(D^{t}\left[\sum_{k=1}^{\# L G S} C_{n k}^{-1}\right] D+C_{\phi}^{-1}\right)^{-1} D^{t}\left[\sum_{k=1}^{\# L G S} C_{n k}^{-1}\right] s_{k}
$$

\subsection{Slope noise covariance matrix and turbulence priors}

For one LGS channel $k$, the slope noise covariance matrix $C_{n k}$ is built accordingly to the method presented in [1] and uses the analytical noise variances of WCoG [4]. We checked the analytical expressions of WCoG give the same results as numerical simulations of SH images for a wide range of $\mathrm{Nph}=250$ phe- up to 1000 phe- (see fig. 5 in [1]). Using all LGS channels, we build a block diagonal noise covariance matrix with $C_{n k}$ blocks. Note we assume all LGS emit the same flux. Assuming that each LGS gives the same elongation pattern, the blocks are the same in case of centre launching and differ from one another in case of edge launching. Since $C_{n k}$ is very sparse we have optimized its inversion to speed up the reconstruction methods. In addition we build the a priori covariance matrix $C_{\phi}$ of the KL wavefront which is diagonal. Being very sparse, its inversion is also optimized for the MAP reconstruction.

\subsection{Geometry}

A full simulation of the modal reconstruction with a $42 \mathrm{~m}$ telescope using a 6 LGS WFS configuration is not operational for the moment. So we have used the code for a smaller but representative geometry. We represent any down-scale observation with a lower diameter telescope by reducing the number of subapertures (i.e. the number of modes) by a factor of $\mathrm{N}$ being the ratio between $42 \mathrm{~m}$ and the lower diameter. In addition we reduce the LGS altitude and the thickness of LGS emission with the same factor. In any events the telescope has a 0.3 central obscuration and the subaperture diameter is $0.5 \mathrm{~m}$. The Fried diameter is kept constant since the turbulence is identical vertically. The LGS emits a plane wave and we consider a Gaussian Sodium profile.

A very small telescope diameter, say $4.2 \mathrm{~m}$, was useful for tests during the code development as presented in section 2.3. Next, to be closer to the real geometry of ELT wide-field-of-view adaptive optics systems, we model a $21 \mathrm{~m}$ telescope. Considering $\mathrm{N}=2=42 \mathrm{~m} / 21 \mathrm{~m}$, we then put the LGS at an altitude of $45 \mathrm{~km}$ instead of $90 \mathrm{~km}$. The thickness of Na emission (actually $10 \mathrm{~km}$ ) is down-scaled to $5 \mathrm{~km}$. The spot elongation at the telescope pupil edge is the same as for a $42 \mathrm{~m}$ telescope. We will consider 6 LGS regularly spaced. The SH WFS has $42=84 / \mathrm{N}=2$ subapertures across telescope diameter and we reconstruct as many modes as those given by the radial order corresponding to $42-1$.

\subsection{Results for a "pupil-only turbulence" case}

First steps in propagation of multi-LGS slope noise have considered as M. Tallon [3] a "GLAO-like" configuration, alias 3-LGS with pupil-only turbulence and without fratricide effect. We set all the parameters given in section 3 of this reference to compare rapidly various phase estimators: LS, WLS, UNIFMAP and MAP. The metric we share here with [3] is the wavefront error in nm (WFE) at center 

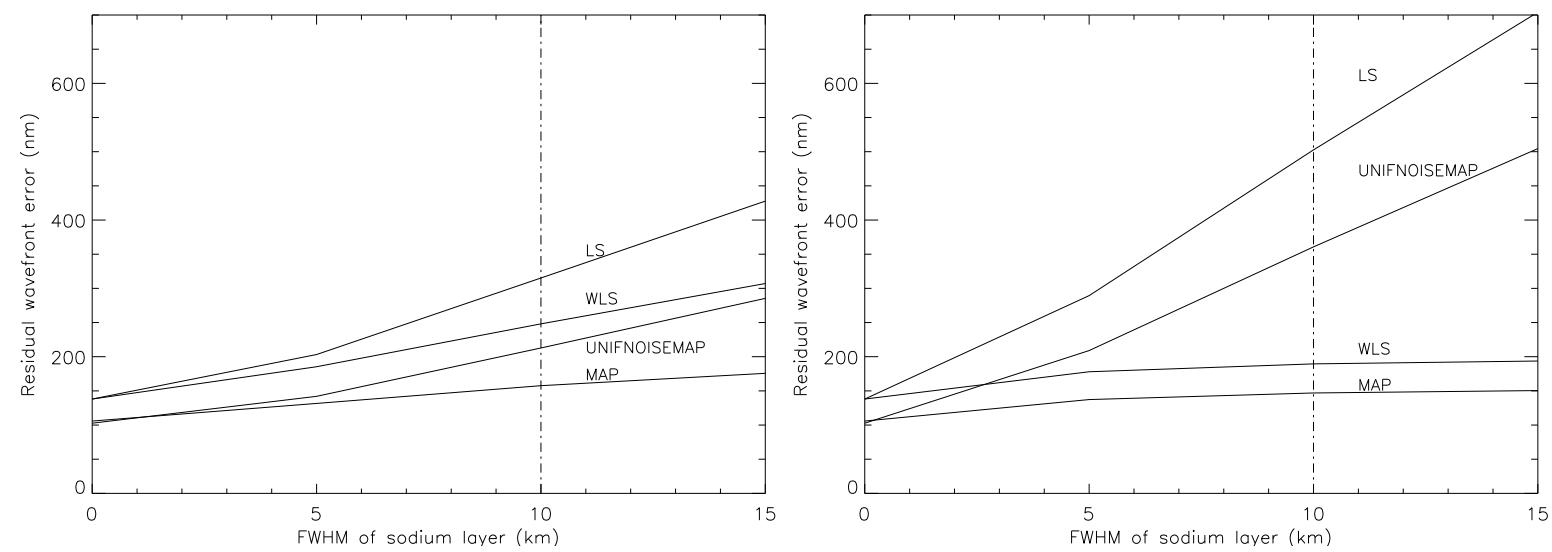

Fig. 1. Propagation of multi-LGS slope noise in a "GLAO" configuration. We compare the wavefront errors using the different reconstruction methods as a function of the LGS elongation. Three LGS are launched on a ring of 2 arcmin radius from the center (on the left) and from the edge (on the right) of the pupil.

FoV which analytically corresponds to variance + squared bias. We present results obtained in a downscaled case $4.2 \mathrm{~m}$ after having applied certain scaling factor deduced from some evaluations with a 42 $\mathrm{m}$ telescope case. We show similarly to [3] that MAP is the optimal LGS WFSing and elongation is no more a problem for edge launching. Moreover the WFE flattens out from a certain elongation let say from $7 \mathrm{~km}$, meaning that long axis slope on very elongated spots could be probably discarded. Then a limitation in the $\mathrm{SH}$ FoV, truncating the spot in the elongated direction, should be investigated to reduce the detector size. We will see in the following the advantage of the edge launching configuration is kept in a full tomographic scheme.

\section{Impact of launching scheme: Central vs edge launching}

In the remaining of the paper we use only the MAP estimator to reconstruct the wavefront. We start from a realistic 9 layer turbulence profile from European Southern Observatory (ESO) that is degraded to 3 equivalent layers, considered as the true turbulence profile. The relative weights are obtained by some under-sampling of the original profile. We observe at Zenith with seeing $=0.8$ arcsec. We thus reconstruct the phase on these 3 layers, the altitudes of which are 0,1106 and $10810 \mathrm{~m}$. We consider here a down-scaled $21 \mathrm{~m}$ telescope diameter and a MAORY-like LGS constellation diameter of 2 arcmin. Other conditions are: Central obscuration: 0.3, number of subapertures in pupil diameter: 42 , noise at subaperture level without elongation: $0.6 \mathrm{rad}^{2}$ at $589 \mathrm{~nm}, \mathrm{Nph}=500$ phe-, $\mathrm{RON}=3 \mathrm{e}-$. Here we consider neither spider nor fratricide effects.

Figure 2 presents similar tomographic WFE for both launching schemes: edge (around M1) and central (behind M2). Even with elongation, noise propagation still resembles the Rigaut-Gendron propagation law with its asymptotic behaviour. Although at very high frequency the propagation law deviates from this due to the regularization brought with the MAP. Tomographic WFE with elongation rises against without elongation by a factor of 3 in variance (1.7 in rms values). This is general for MAORY/ATLAS/EAGLE projects with every LGS asterism. Looking in details, LGS elongation still induces a specific modal noise signature with excited modes. This is specially obvious for the central launching. Edge launching gives smoother propagation onto modes, closer to the case without elongation apart from a global multiplicative factor. This is rather consistent with the notion of a slope equivalent uniform noise used in Fourier simulations (see section 6). 


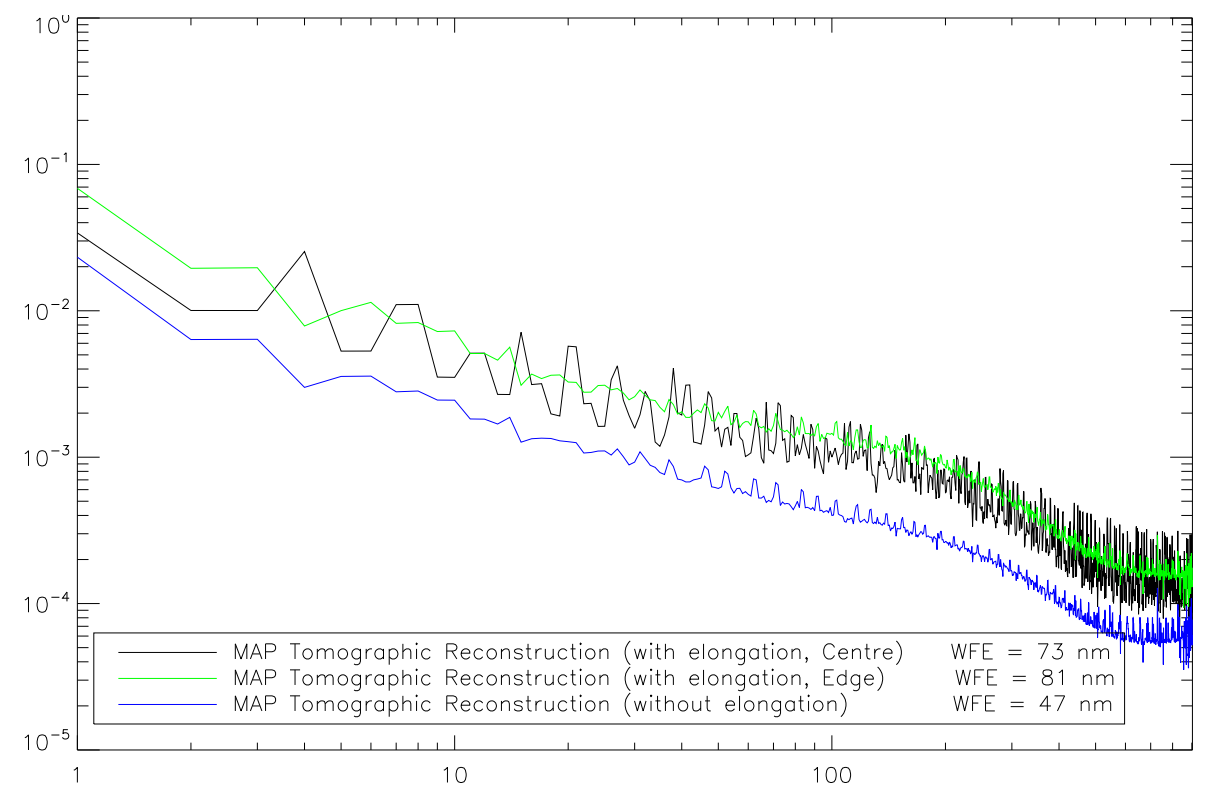

Fig. 2. Comparison of tomographic performances in case of a $21 \mathrm{~m}$ half-scaled telescope between central $(\mathrm{WFE}=73 \mathrm{~nm}$ ) and edge launching $(\mathrm{WFE}=81 \mathrm{~nm})$. Fratricide and spider effects are excluded. For comparison the ideal non-elongated case $(\mathrm{WFE}=47 \mathrm{~nm}$ ) is also plotted.

\section{Impact of fratricide effect}

Systems using multiple LGS beams with CW lasers are affected by fratricide effect, related to Rayleigh or Mie scattering of the laser light in the lower atmosphere. Here we consider only Raleigh scattering on the air molecules. A WFS looking at a LGS may collect the scattered light of other LGSs, causing a background increase in some subapertures. This is true when the LGSs are projected from behind M2 for instance, while in the case of edge launching around M1 the effect is not present. In this section we perform an impact analysis of the fratricide effect on the tomographic WFE considering the three projects MAORY, ATLAS, EAGLE. To do so, we assume the contribution of the Rayleigh background translates into an additional uniform noise in the affected subapertures.

We assume that each LGS gives the same maximum number of Rayleigh photons in a subaperture FoV of $9 \times 9$ arcsec. The "absolute" flux of the Rayleigh background is consistent with a Na return flux of approximately 500 detected photons per subaperture per LGS and per frame and a $8 \mathrm{~W}$ laser power. About $15 \%$ of the sub-apertures present an integrated fratricide background that is higher than the Na return flux. Note each project has a peculiar LGS constellation diameter (see Table 1). That leads to slightly different patterns of Rayleigh light as shown on Figure 3 from top to bottom. In fact we get the slowest decrease of Rayleigh flux across the pupil in the EAGLE-like case (bottom panel).

Based on these elements, we use our modal LGS tomographic code for MAORY, ATLAS, EAGLE projects in the same way we did in section 3. We again consider a 3 equivalent-layers turbulence profile deduced from the 9 layer profile from ESO and with 0.8 arcsec seeing. Moreover we envisage 500 phe-/subap/frame and a RON of 3 electrons. Tomographic WFE performance is evaluated for the two launching schemes. When removing the fratricide effect for the central launching, the global tomographic reconstruction leads to a slight advantage in performance for central launching option. When including fratricide effect, results in Table 1 show that edge launching leads to better performance for MAORY, ATLAS but EAGLE. However the advantage of edge launching is regained for EAGLE when more layers are reconstructed as shown in section 5. Based on these results one would tend to prefer the edge launching, for better performance and also to limit risks associated with a possible underestimation of fratricide scatter. 


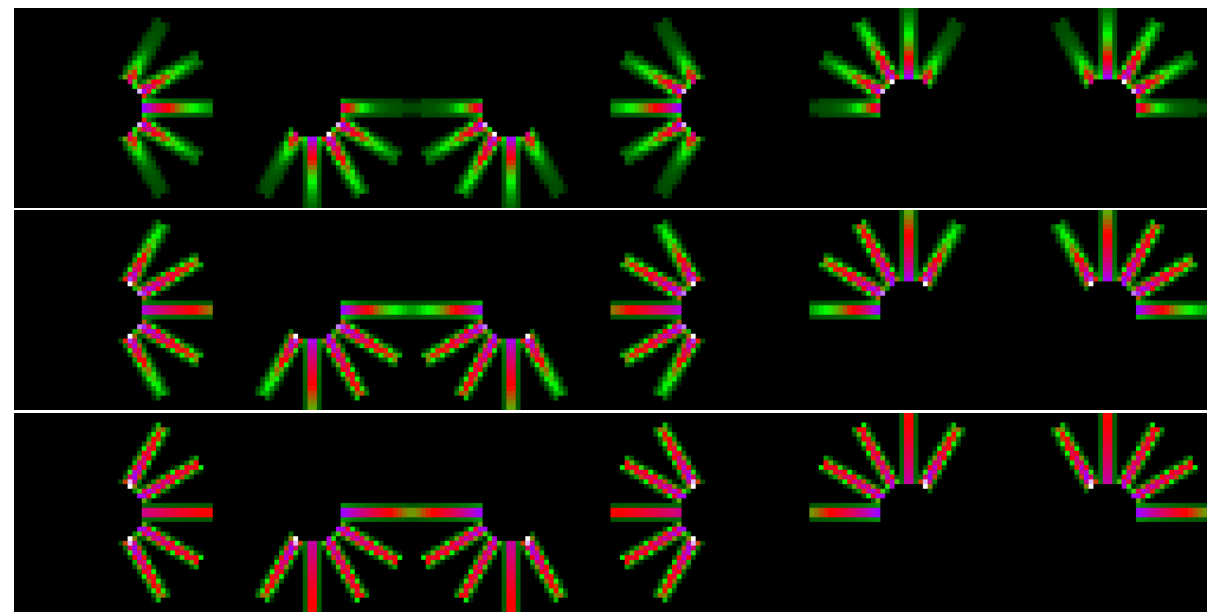

Fig. 3. Examples of fratricide effects on $21 \mathrm{~m}$ downscaled cases: MAORY-like (top), ATLAS-like (medium), EAGLE-like (bottom). The 6 images side by side give the fratricide background pattern for each of the 6 LGS. Subapertures affected by Rayleigh scattering in the pupil plane of one WFS looking at one LGS.

Table 1. WFE for the different projects and both launching schemes, and comparisons in quadratic difference.

\begin{tabular}{lccc}
\hline LGS constellation & MAORY & ATLAS & EAGLE \\
diameter & 2 arcmin & 4.2 arcmin & 7.2 arcmin \\
\hline Centre (witout fratricide) & $73 \mathrm{~nm}$ & $87 \mathrm{~nm}$ & $111 \mathrm{~nm}$ \\
Centre (with fratricide) & $92 \mathrm{~nm}$ & $111 \mathrm{~nm}$ & $145 \mathrm{~nm}$ \\
Amount due to fratricide & $+56 \mathrm{~nm}$ & $+69 \mathrm{~nm}$ & $+93 \mathrm{~nm}$ \\
Edge & $81 \mathrm{~nm}$ & $108 \mathrm{~nm}$ & $166 \mathrm{~nm}$ \\
Edge vs. Centre (with fratricide) & $-44 \mathrm{~nm}$ & $-26 \mathrm{~nm}$ & $+80 \mathrm{~nm}$ \\
\hline
\end{tabular}

\section{Impact of the number of turbulent layers}

The impact of profile uncertainties and of number of layer to reconstruct is probably of concern for the largest LGS asterisms. Before that, we study the influence of varying in the same way the number of true/reconstructed layers of the $C_{n}^{2}$ profile. For EAGLE for instance, we again model a down-scaled but representative observation with a $21 \mathrm{~m}$ telescope diameter. We here consider $\mathrm{Nph}=500$ phe- per subaperture per frame, a read out noise $\mathrm{RON}=3$ electrons and a seeing equal to 0.8 arcsec. At this stage we include fratricide effect on central launching. We compute the modal WFE for a 3 layer profile the altitudes of which are: $0 \mathrm{~m}, 1106 \mathrm{~m}$ and $10810 \mathrm{~m}$. We compute also the modal WFE for a 5 layer profile. The corresponding altitudes are: $0 \mathrm{~m}, 140 \mathrm{~m}, 281 \mathrm{~m}, 562 \mathrm{~m}$ and $6391 \mathrm{~m}$. In this last case note the layer altitudes are lower and hence present larger footprint overlap. The results in term of WFE are shown in Table 2 for the different cases. First, one can see that the WFE values are lower with 5 layers compared with with 3 layers. This may come from the overlap which is more favourable in the 5 layers case. Second the global tomographic reconstruction leads equivalent performance for both launching options with 5 layers.

Table 2. WFE varying the number of true=reconstructed layers for an EAGLE-like case at low LGS return flux.

\begin{tabular}{lrr}
\hline WFE $(\mathrm{nm})$ & 3 layers & 5 layers \\
\hline Centre (with fratricide) & $145 \mathrm{~nm}$ & $112 \mathrm{~nm}$ \\
Edge & $166 \mathrm{~nm}$ & $112 \mathrm{~nm}$ \\
No elongation & $77 \mathrm{~nm}$ & $62 \mathrm{~nm}$ \\
\hline
\end{tabular}




\section{LGS WFS noise for Fourier code}

The Fourier code [9] we use for point spread function predictions does not account for LGS elongation, since it considers only uniform slope noise on all subapertures. To do so we define a "slope equivalent uniform noise" per subaperture at $589 \mathrm{~nm}$, which is equivalent to the tomographic WFE calculated with elongation. Now we have selected the baseline launching option we can evaluate this uniform slope noise variance (this corresponds to the non elongated case with amplified noise) so that the global WFE is equal to the edge launching WFE. In MAORY configuration we find a $2 \mathrm{rad}^{2}$ equivalent noise (factor 3 amplification compared to standard non elongated $0.6 \mathrm{rad}^{2}$ ). Equalizing WFE also leads to a fairly good adequation of WFE modal distribution. The equivalence would not be so perfect for central launching since the modal signature is in this case more contrasted and quite different from a uniform noise case. Finally when $\mathrm{Nph}=500$ phe-/frame/subaperture, commonly for ATLAS and for EAGLE, the slope equivalent noise amounts respectively to $2 \mathrm{rad}^{2}$. This figure is possibly multiplied by a noise filtering factor induced by the AO loop and differs from values considered in Phase A projects studies.

\section{Conclusion and perspectives}

We have developed a tomographic modal (KL) reconstruction pseudo-analytical tool giving an estimation of the WFE in the FoV using multi-channel LGS WFS data. This estimation is actually a common part to all wide FoV AO concepts. It depends on GS characteristics (geometry, flux) and of course on the kind of estimator. We show that simple least-square estimators must be dropped since more sophisticated approach such as MAP brings a significant gain. Of course with a MAP approach we need priors on turbulence statistics and its distribution in altitude. Our analysis of the tomographic wavefront reconstruction with a half-scaled telescope of $21 \mathrm{~m}$ diameter shows that spot elongation leads to a noise variance excess which amounts 3 times the case without elongation regarding edge launching. Consequently, laser power requirements should be moderate based on just improved today technology. Therefore pulsed laser solutions that reduce spot elongation are less mandatory. Finally when including fratricide effect due to Rayleigh scattering, slightly better performance is obtained with edge launching.

This analysis could be pursued to get closer to ELT $42 \mathrm{~m}$ configuration. First compare our modal reconstruction to the zonal one. Both are expected to give the same performance but the latter being more computing efficient should refine results for a truly $42 \mathrm{~m}$ case. Second account for spherical waves for actual ELT geometry. A detailed analysis of LGS WFS with spot elongation and Sodium profile variations should bring reasonable detector characteristics. Of course the impact of $C_{n}^{2}$ model errors has to be analyzed and specially the exact number of layers to be reconstructed.

\section{Acknowledgments}

This work was supported by the European Commission, Seventh Framework Programme (FP7), Capacities Specific Programme, Research Infrastructures; specifically the FP7, Preparing for the Construction of the European Extremely Large Telescope Grant Agreement, Contract number INFRA2007-2.2.1.28. This work also received the support of ESO and PHASE, the high angular resolution partnership between ONERA, Observatoire de Paris, CNRS and Université Paris Diderot. The authors are very grateful to L. Schreiber and E. Diolaiti for their contribution to reading the draft and insight commentary. The authors thank also Anne Costille, Jean-François Sauvage, N. Muller and G. Rousset for fruitful exchanges they have with them.

\section{References}

1. Laura Schreiber et al., MNRAS Vol. 396, Issue 3, (2009) pp. 1513-1521

2. Clélia Robert et al., JOSA A Vol. 23, Issue 3, (2006) pp. 613-624

3. Michel Tallon et al., Adaptive Optics Systems Proceedings of SPIE Vol. 7015, (2008)

4. Sandrine Thomas et al., MNRAS Vol. 387, Issue 1, (2008) pp. 173-187

5. N. Muller et al., 1st AO4ELT conference Paris (2009)

6. E. Diolaiti et al., 1st AO4ELT conference Paris (2009)

7. T. Fusco et al., 1st AO4ELT conference Paris (2009)

8. G. Rousset et al., 1st AO4ELT conference Paris (2009)

9. B. Neichel et al., JOSA A Vol. 26, Issue 1, (2009) pp. 219-235 